\title{
NONLOCAL ANALYSIS OF MODULAR ROLES
}

\author{
J. M. BULDÚ , I. SENDIÑA-NADAL, I. LEYVA \\ and J. A. ALMENDRAL
}

M. ZANIN

S. BOCCALETTI

\begin{abstract}
We introduce a new methodology to characterize the role that a given node plays inside the community structure of a complex network. Our method relies on the ability of the links to reduce the number of steps between two nodes in the network, which is measured by the number of shortest paths crossing each link, and its impact on the node proximity. In this way, we use node closeness to quantify the importance of a node inside its community. At the same time, we define a participation coefficient that depends on the shortest paths contained in the links that connect two communities. The combination of both parameters allows to identify the role played by the nodes in the network, following the same guidelines introduced by Guimerà et al. [Guimerà \& Amaral, 2005] but, in this case, considering global information about the network. Finally, we give some examples of the hub characterization in real networks and compare our results with the parameters most used in the literature.
\end{abstract}

Keywords: Complex networks; community role; betweenness.

\section{Introduction}

During the last years, complex network analysis has given fruitful information about complex systems from a new perspective [Newman, 2003; Boccaletti et al., 2006; Costa et al., 2011], where the structure of the network constrains the dynamical processes occurring in it and, at the same time, the dynamics of the nodes can influence the evolution of the network structure [Gross \& Blasius, 2008]. One of the advantages of this new perspective is 
that we are able to analyze not only the network as a global entity but the role that nodes play inside it. Moreover, we have to consider that real networks have certain modularity, which is related to the appearance of community structures that are crucial in the dynamical processes taking place on top of the network [Almendral et al., 2011]. Therefore, the interplay between the individual nodes (microscale), the existing communities (mesoscale) and the behavior of the whole network (macroscale) can only be understood from a multilevel approach with different scales interacting with each other.

Within this framework, Guimerà et al. [Guimerà \& Amaral, 2005] introduced a twodimensional characterization regarding the importance of each node inside its own community and the connection with other communities of the network. Specifically, they defined a within-module degree $z_{i}$ and a participation coefficient $p_{i}$, the former taking into account the importance of a node inside its own community and the latter measuring how diverse are the links that are sent to other communities in the network. The methodology of Guimerà et al. [2005] has been applied to a wide manifold systems such as social [Teitelbaum et al., 2008; Moon et al., 2010], technological [Guimerà et al., 2005; Costa et al., 2007] and biological networks [Guimerà \& Amaral, 2005; Hagmann et al., 2008; Buldú et al., 2011]. More recently, Arenas et al. [2010] have defined different indicators to evaluate the role of the nodes of a network. In this case, authors took advantage of the singular value decomposition of the participation matrix, which contained the information regarding how a node spreads its connections among the communities of the network.

Despite being good approaches for evaluating the role of the nodes, both methods are local in scope, since they only take into account the origin and final community of the links, no matter what nodes are being attached to. In this way, links that connect different community hubs and those connecting collateral nodes end up with the same relevance, which may have important implications, specially in the computation of the participation coefficient. Looking back, it is not the first time that disregarding the importance of links has led to counterintuitive results. This is the case of the heterogeneity paradox [Nishikawa et al., 2003], where a small-world topology that reduces the number of steps between nodes, seemed to be inadequate to achieve synchronization between nodes. The heterogeneity paradox was solved in parallel by Motter et al. [2005] and Chavez et al. [2005] by assigning weights to the links according to their degree [Motter et al., 2005] or their betweenness [Chavez et al., 2005]. Therefore, the introduction of weights in the links was reflected in differences between relevant and nonrelevant connections, leading to an increase of the network synchronizability.

In the current work, we are concerned about how to assign weights to the links of a network in order to better identify the role that a node is playing in the community structure. With this aim, we propose a new method to evaluate the participation of the nodes in their neighboring communities and redefine the importance of a node inside its community in terms of the community closeness. Following the ideas introduced by Chavez et al. [2005], we propose the use of the link betweenness (more precisely, the number of shortest paths) in order to weigh the relevance of the inter-community links and, therefore, the participation coefficient $p_{i}^{b}$ of the nodes. Next, we define a $z$-score $z_{i}^{c}$ based on the proximity of a node to its neighbors in the community. Both parameters allow to identify the provincial and connector hubs [Guimerà \& Amaral, 2005] using global information about the network structure, a fact that was disregarded in the methods previously reported. Finally, we check the application of the proposed method by analyzing the structure of four real networks, with special attention to the discrepancies in the role assignment provided by the classical method [Guimerà \& Amaral, $2005]$.

\section{Analysis of Roles}

The role assignment introduced by Guimerà et al. [Guimerà \& Amaral, 2005] departs from a partition of a network into communities. Network's partition must be already known from experimental observations or obtained by applying one of the many existing community detection algorithms [Fortunato, 2010]. Once the community structure is known, we have to go down to the lowest scale (i.e. node level) in order to analyze the role of the nodes in their corresponding communities. The classical classification of nodes is based on the computation of the within-module degree $z_{i}$ (also known as $z$-score) and the participation coefficient $p_{i}$. The former parameter quantifies the importance of the 
node $i$ inside its community and it is defined as:

$$
z_{i}=\frac{k_{i}-\left\langle k_{\mathrm{com}_{i}}\right\rangle}{\sigma_{k_{\mathrm{com}_{i}}}}
$$

where $k_{i}$ and $\mathrm{com}_{i}$ are, respectively, the degree and the community of the node $i,\left\langle k_{\mathrm{com}_{i}}\right\rangle$ is the mean degree of the community $\operatorname{com}_{i}$ and $\sigma_{k_{\mathrm{com}_{i}}}$ is the standard deviation of $k$ in $\operatorname{com}_{i}$. The $z$-score of a node is zero if it has a degree $k$ equal to the average of the community. Positive (negative) values of $z_{i}$ reveal that the node has more (less) connections than the average. On the other hand, the participation coefficient $p_{i}$ indicates how connections of the node $i$ are distributed among the existing communities:

$$
p_{i}=1-\sum_{j=1}^{N_{\mathrm{com}}}\left(\frac{k_{i}^{\mathrm{com}_{j}}}{k_{i}}\right)^{2}
$$

where $k_{i}^{\mathrm{com}_{j}}$ is the number of connections of node $i$ that go into community $\operatorname{com}_{j}$ and $N_{\text {com }}$ is the total number of communities. The participation coefficient is zero when all links of a node are inside its community and close to one when they are distributed among all modules of the network.

Figure 1(a) shows an example with a test network (Test Network A) where both parameters have been calculated. The network is divided into four communities and it has three kinds of nodes: (a) connector hubs (1, 17, 33 and 49), which are relevant nodes inside their communities, (b) nonhub connectors $(2,3,18,19,34,50$ and 51), which are nodes with low relevance in their communities, but with connections to other communities, and (c) peripheral nodes (the rest), which are not relevant inside and outside their communities. The upper inset of Fig. 1(b) shows the values of $z_{i}$ and $p_{i}$ for each kind of node. Interestingly, we can observe how nonhub connectors have a higher participation coefficient than the connector hubs, despite hubs being connected to the hubs of other communities. This is a consequence of having a high number of connections with nodes of their own community and the fact that each link has the same weight in the participation coefficient. Nevertheless, if we compute the number of shortest paths that cross each link of the network [see Fig. 1(b)], we observe that links connecting to hubs have a higher number of shortest paths passing through them, which should be reflected in a higher participation coefficient.

To overcome this drawback we need to redefine the participation coefficient of a node and, in addition, its community $z$-score. With this aim, we follow the guidelines given in [Chavez et al., 2005] and weigh each link according to the number of shortest

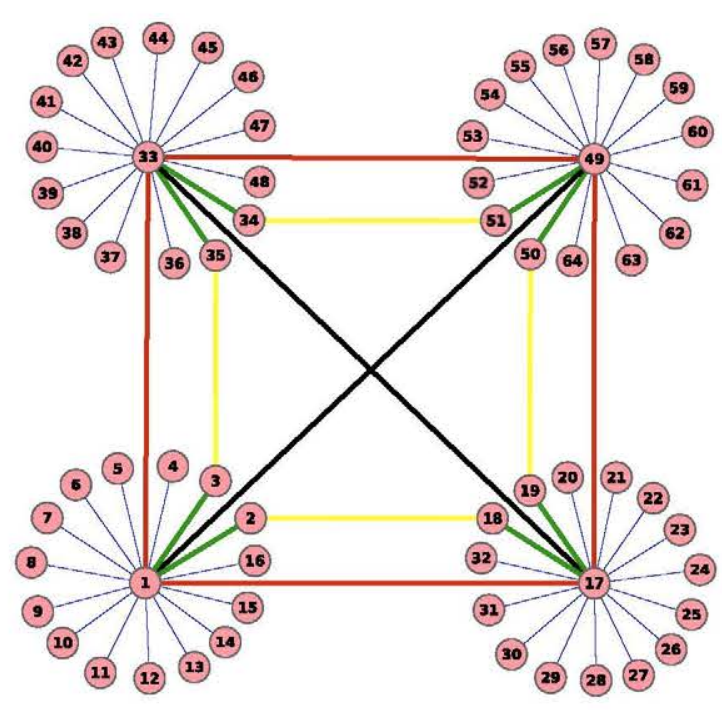

(a)

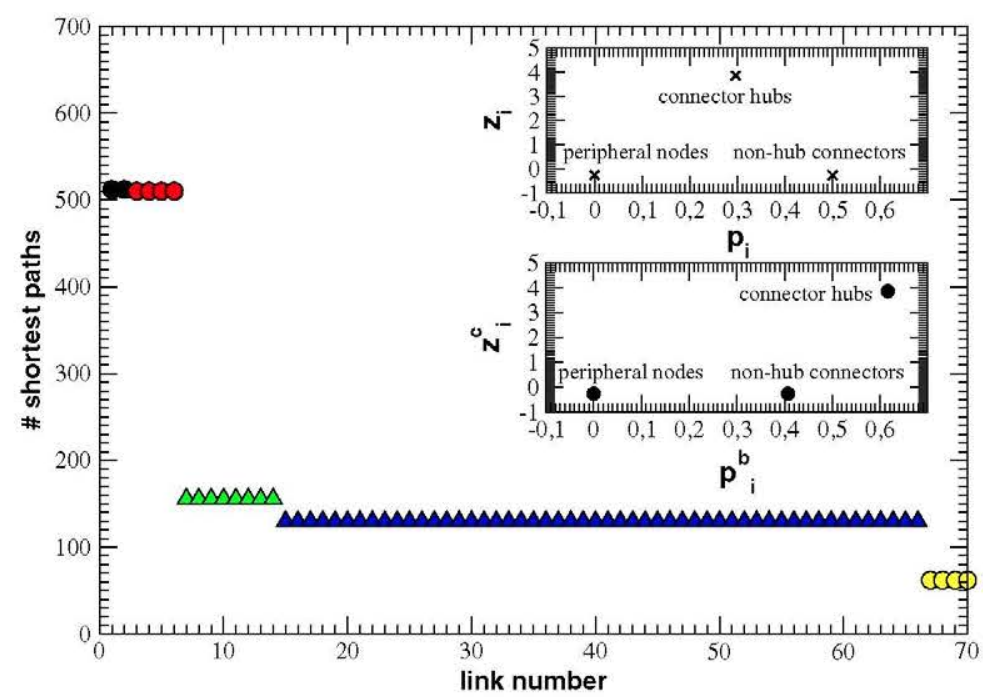

(b)

Fig. 1. (a) Structure of Test Network A, which is split into four communities. According to the number of links, there are three kinds of nodes: (1) connector hubs (1, 17, 33 and 49), (2) nonhub connectors (2, 3, 18, 19, 34, 35, 50 and 51) and (c) peripheral nodes (the rest). Colors have been assigned according to the different kind of links: (1) red and black, links between community hubs, (2) yellow and green, links between nonhub connectors and between nonhub connectors and peripheral nodes, respectively, and (3) blue, internal links. (b) Plots of the number of shortest paths crossing each kind of link and the $\left(p_{i}, z_{i}\right)$ and $\left(p_{i}^{b}, z_{i}^{c}\right)$ plots for the three kinds of nodes, calculated as in [Guimerà \& Amaral, 2005] (upper inset) and with our proposed metrics (bottom inset). 
paths that go through it. Now, the importance of the node $i$ inside its community is quantified with a $z$-score based on its community closeness $c_{i}^{\text {in }}$, which is the inverse of the minimum number of steps that we have to make in order to go from node $i$ to any other inside its community:

$$
c_{i}^{\text {in }}=\frac{1}{k_{i}^{\text {in }}} \sum_{j \in V_{i}} \frac{1}{d_{i j}}
$$

where $k_{i}^{\text {in }}$ is the degree of node $i$ inside its community, $V_{i}$ is the set of community neighbors of node $i$, and $d_{i j}$ is the shortest distance between nodes $i$ and $j$. Hence, we define the community relevance $z_{i}^{c}$ as the $z$-score of the node closeness:

$$
z_{i}^{c}=\frac{c_{i}^{\mathrm{in}}-\left\langle c_{\mathrm{com}_{i}}^{\mathrm{in}}\right\rangle}{\sigma_{c_{\mathrm{com} i}^{\mathrm{in}}}}
$$

where $\left\langle c_{\mathrm{com}_{i}}^{\mathrm{in}}\right\rangle$ is the average closeness of the community $\operatorname{com}_{i}$ and $\sigma_{c_{\mathrm{com}}^{\mathrm{in}}}$ is the standard deviation of $c^{\text {in }}$ inside $\operatorname{com}_{i}$. Figure 2 shows an example (Test Network B) of how a community relevance measure based on closeness can better quantify the importance of a node inside its community. In this case, the network has a unique community of fifteen nodes, with two hubs (nodes 1 and 9) and one connector node inside the community (node 8 ). Note that all nodes, apart from the hubs, have degree two, which is reflected in the same $z$-score $z_{i}$ when it is based on the node degree. Nevertheless, we would disregard the importance of node 8 in the transmission of information inside the community, since all shortest paths between nodes $1-7$ to nodes 9-15 pass through it. Therefore, a $z$-score based on closeness enhances the relevance of connector nodes, such as node 8, as we can see in Fig. 2(b) where $z_{i}$ and $z_{i}^{c}$ are compared. With $z_{i}^{c}$, the importance of node 8 increases when compared with other nodes of the same degree and, at the same time, it is still lower than the $z_{i}^{c}$ of the network hubs.

With regard to the relevance in the connection between communities, we define the betweenness participation coefficient $p_{i}^{b}$, which quantifies how the shortest paths that pass through the links of a node $i$ are distributed among the existing communities:

$$
p_{i}^{b}=1-\sum_{j=1}^{N_{\mathrm{com}}}\left(\frac{B_{i}^{\mathrm{com}_{j}}}{B_{i}}\right)^{2}
$$

where $B_{i}^{\mathrm{com}_{j}}$ is the number of shortest paths that pass through those links of node $i$ that fall into

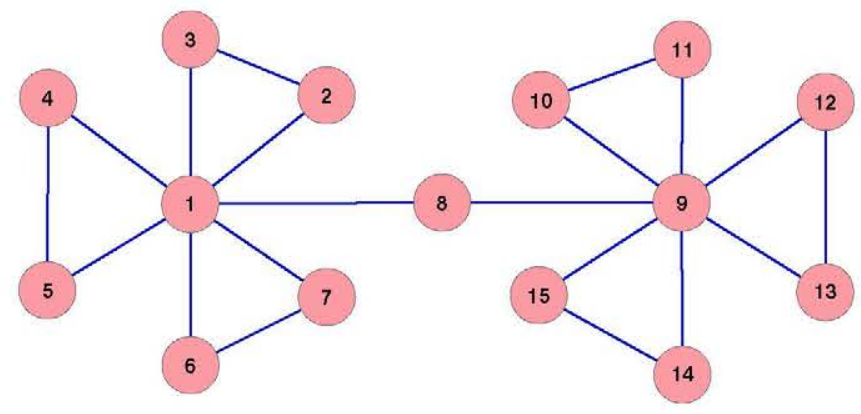

(a)

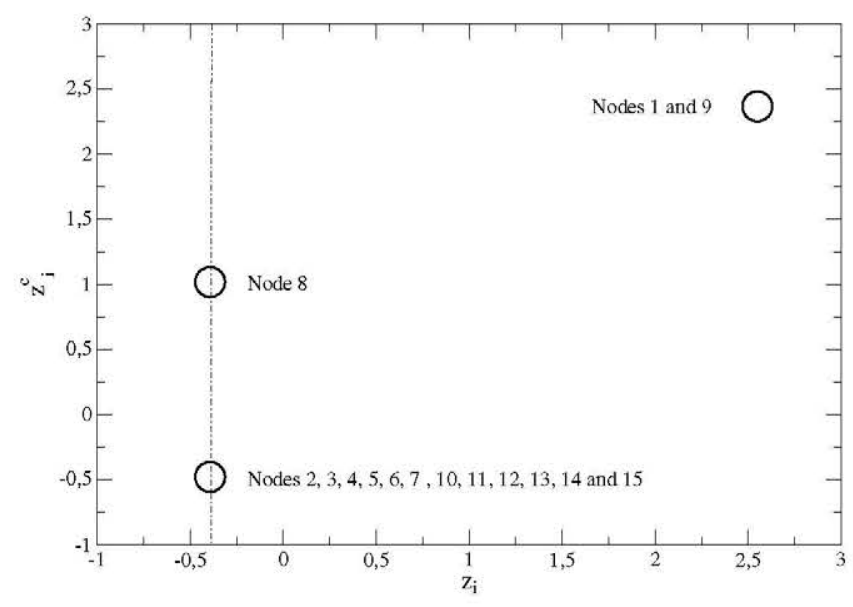

(b)

Fig. 2. (a) Structure of Test Network B. In this example, we assume that all nodes belong to the same community. Nodes 1 and 9 are the community hubs, with a degree $k_{\text {hub }}=7$, while the rest of the nodes have the same degree $k_{\text {nonhub }}=2$. Note that node 8 , despite having the same number of connections as the other nonhub nodes, is a relevant node, since it connects the left and right sides of the community. (b) Plot showing $z_{i}$ versus $z_{i}^{c}$. We can see that a measure of the $z$-score that is based on closeness $\left(z_{i}^{c}\right)$, allows to differentiate among nodes of the same degree, but with different relevance.

community $\operatorname{com}_{j}, B_{i}$ is the total number of shortest paths that require a link that is attached to $i$ and $N_{\text {com }}$ is the total number of communities. The betweenness participation coefficient $p_{i}^{b}$ is zero when all links of a node $i$ are inside the same community or in the absence of shortest paths crossing node $i$. On the contrary, it is close to one when links of node $i$ contain shortest paths equally distributed among all modules of the network. The inset of Fig. 1(b) shows how the new participation coefficient promotes the impact of nodes with higher betweenness, which are now the nodes with higher participation $p_{i}^{b}$ in the network. Due to the simplicity of the Test Network A, we do not observe differences in the 
community relevance $z_{i}^{b}$, despite them appearing for more complex community structures.

\section{Results}

\subsection{Characterizing community hubs}

In order to test the power of our method, we analyze four real networks of different nature and size [Almendral et al., 2010], namely, the Zachary karate club [Zachary, 1977], a dolphin network [Lusseau \& Newman, 2004], a network of political books in
USA [Krebs, 2011] and the network of domestic flights in the USA [Newman, 2004]. Table 1 summarizes the basic topological parameters of these networks.

As mentioned in the previous section, our method is of special interest when there exist differences in the ability of the links to transmit information, which is measured, in our case, by the number of shortest paths $n_{\mathrm{sp}}(j)$ crossing each connection $j$. Figure 3 shows the ranking of the links with regard to $n_{\mathrm{sp}}(j)$. As we can observe in all four examples, there is a high heterogeneity in the number of shortest paths of each link. This difference in

Table 1. Summary of the parameters of the networks under analysis.

\begin{tabular}{lccccc}
\hline Description & Test Network A & Karate Club & Dolphin Network & Political Books & USA Natl. Airports \\
\hline Nodes (N) & 64 & 34 & 62 & 105 & 332 \\
Links (L) & 70 & 78 & 159 & 441 & 2126 \\
Communities (M) & 4 & 2 & 4 & 3 & 5 \\
Shortest Path (L) & 2.63 & 2.41 & 3.36 & 3.08 & 2.74 \\
Clustering (C) & 0.0012 & 0.554 & 0.255 & 0.483 & 0.620 \\
\hline
\end{tabular}

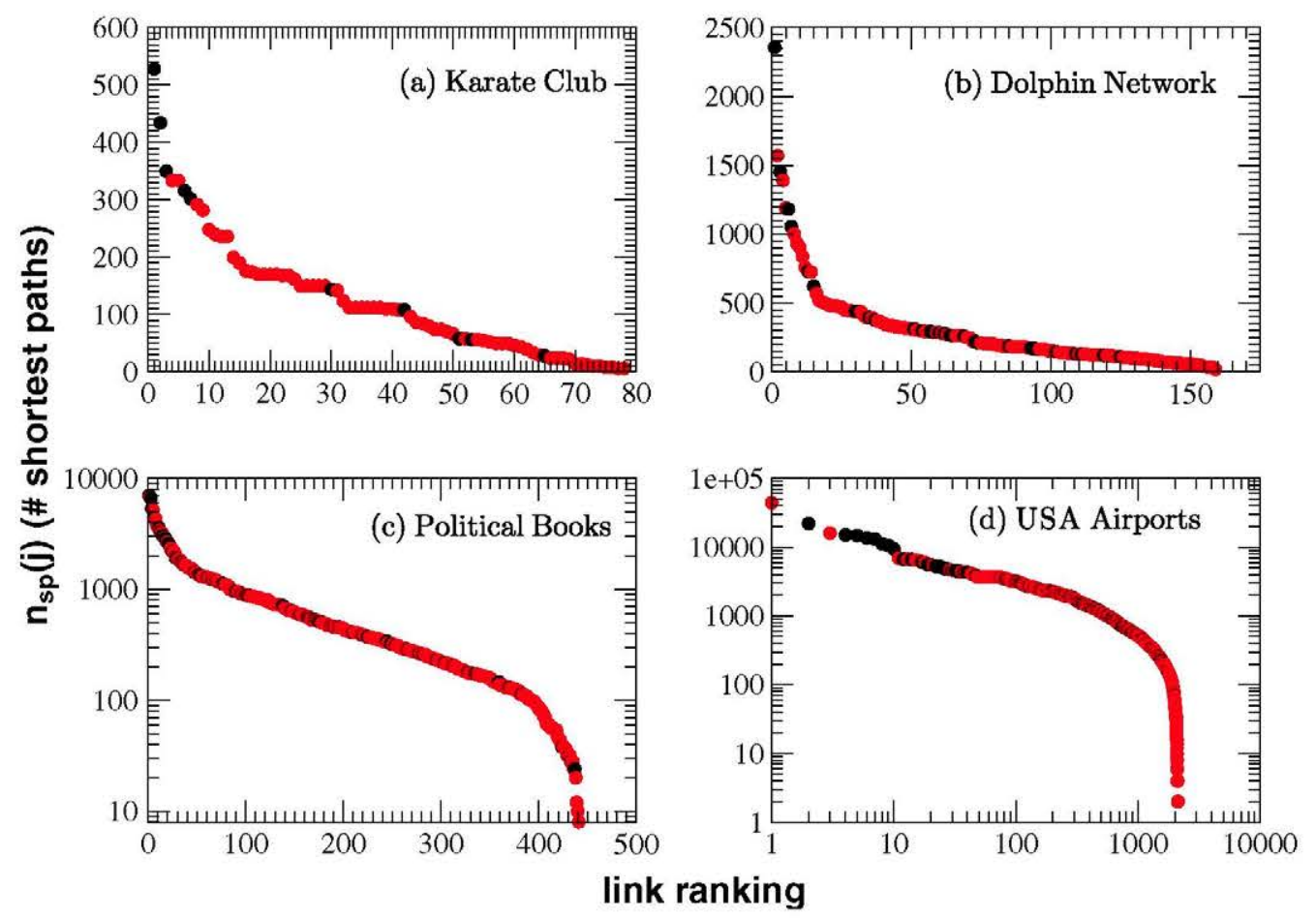

Fig. 3. Link ranking based on the number of shortest paths for four different real networks: (a) Zachary Karate Club, (b) Dolphin Network, (c) USA Political Books in Amazon's web page and (d) National Airport Network of the USA. Note that in all cases there is a clear difference in the number of shortest paths crossing each link, which leads to links with more/less importance. In (a) and (b) we observe a two-slope linear dependence. In (c), intermediate positions of the ranking follow an exponential decay (note the log-linear scale). Finally, the airport network in (d) follows a power law decay with an exponential cut-off for the last nodes of the ranking (in this case, note the $\log -\log$ scale). Links inside and between communities are plotted in red and black, respectively. 
the link importance is particularly displayed in the ranking of the two largest networks, which have, respectively, an exponential decay [book network, Fig. 3(c)] and a power-law decay with exponential cut-off [airport network, Fig. 3(d)].

Once these differences have been observed, we have to check whether the inclusion of the link importance introduces changes in the role played by the nodes in the community structure. Notice that this is an important issue since the calculation of the shortest paths, which includes global information about the network, is much more CPU demanding than any other local measure of the network. With this aim, we calculate (for all networks) the within-module degree $z_{i}$ and participation coefficient $p_{i}$ of each node [Eqs. (1) and (2)] and elaborate a ranking based on both parameters. Next, we compute the community relevance $z_{i}^{c}$ and the betweenness participation coefficient $p_{i}^{b}$ and recalculate the ranking of all nodes with the new parameters. Finally, we analyze the changes observed by comparing both rankings. Figure 4 shows a twodimensional plot of the increase/decrease of the node ranking, both in the relevance inside their communities $\Delta z_{i}=\operatorname{rank}\left(z_{i}^{c}\right)-\operatorname{rank}\left(z_{i}\right)$ and in their participation in the overall community structure $\Delta p_{i}=\operatorname{rank}\left(p_{i}^{b}\right)-\operatorname{rank}\left(p_{i}\right)$. Node sizes are proportional to their number of connections. Figure 4 gives a snapshot on the information gained by the redefinition of the role assignment. The closer a node is to the value $(0,0)$, the less information we gain from the new method. On the contrary, deviations from the origin of coordinates indicate that the number of shortest paths contained in the links of a given node are increasing/decreasing its relevance in the inter- (or intra-) community structure. We can see in Fig. 4 that, although some nodes remain close to the origin, others have strong deviations in one or both parameters. Interestingly, nodes with higher degrees (i.e. indicated by larger radius in the figure) show larger variations in the participation coefficient and not in the intra-community relevance. This is somehow expected and indicates that the importance of a node in their community is mainly dominated by its internal degree (despite there being some exceptions).

In order to have a deeper insight about the information gained by our method, we elaborate

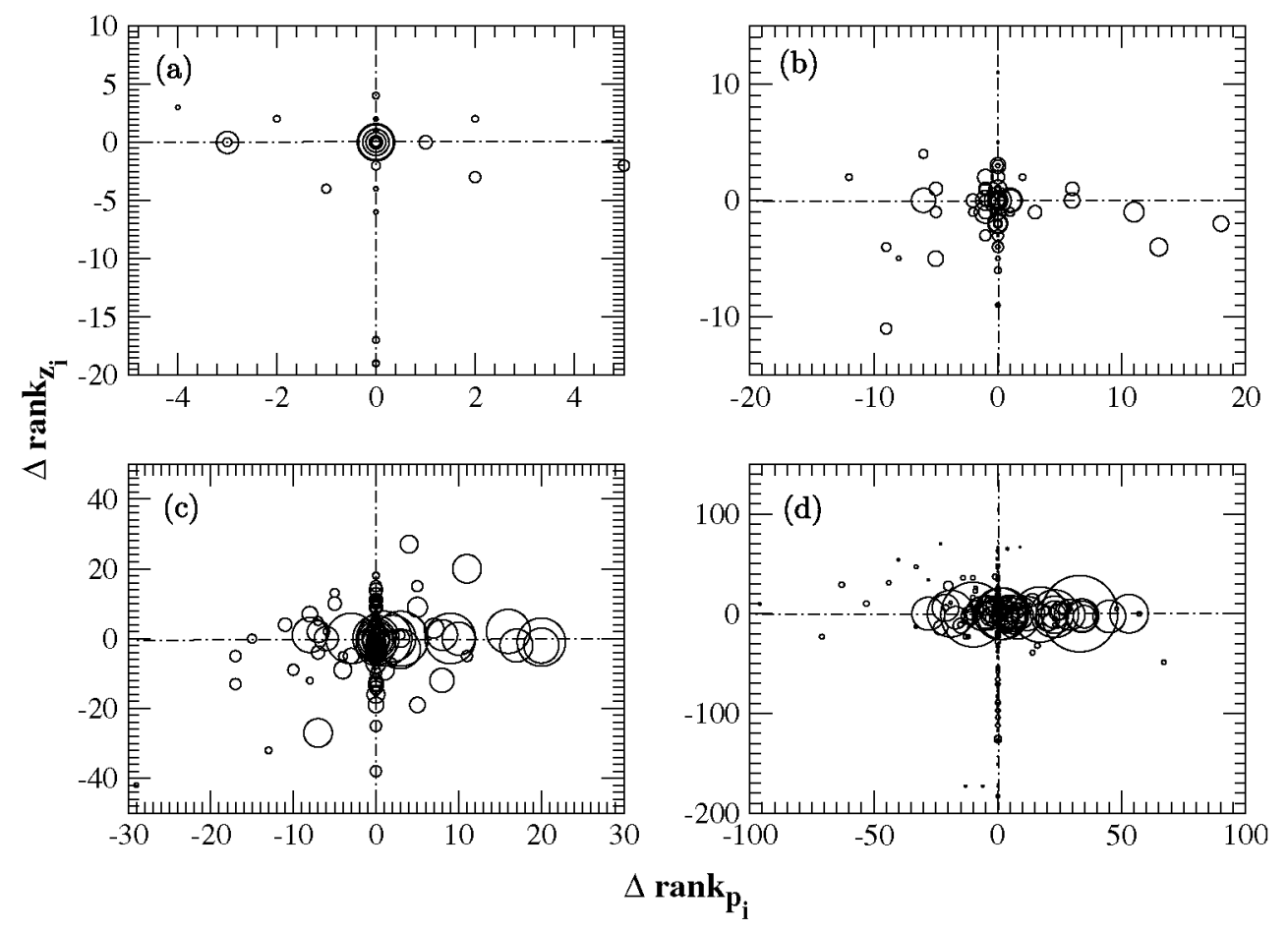

Fig. 4. Two-dimensional plot showing the changes in the ranking of nodes due to the use of role parameters based on shortest path analysis. Networks are the same as those described in Fig. 3: (a) Zachary Karate Club, (b) Dolphin Network, (c) Political books in the Amazon webpage and (d) National Airport Network of the USA. Specifically, we plot $\Delta z_{i}=\operatorname{rank}\left(z_{i}^{c}\right)-\operatorname{rank}\left(z_{i}\right)$ versus $\Delta p_{i}=\operatorname{rank}\left(p_{i}^{b}\right)-\operatorname{rank}\left(p_{i}\right)$, which indicates the reordering in the community relevance and participation, respectively. Node sizes are proportional to their degrees. 
in detail the role variations in the network of airport connections [Newman, 2004; Almendral et al., 2010]. The network is formed by 332 airports and 2126 flight connections. The application of the fast algorithm developed by Newman [2004] provides an optimal partition consisting of a structure of five communities: the largest community $\mathrm{C} 1$ comprising the airports of the west and central parts, and the second largest $\mathrm{C} 2$ grouping the airports from the eastern part. The third community C3 includes airports from southern-east states Louisiana, Mississippi, Alabama and Florida and the fourth community $\mathrm{C} 4$ is for airports in the area of Montana. Finally, airports in Alaska are grouped in the smallest community C5.

Table 2 summarizes the modification of the role of the nodes induced by the new measures of the community relevance and network participation. We observe that the community relevance $z_{i}^{c}$ does not change significantly, which indicates that the use of the node degree in order to evaluate the importance of a node inside its community is a good approach. Nevertheless, there are significant variations in the participation coefficient $p_{i}^{b}$. In this case, there are six new airports that raise to the top-ten ranking when compared with the previous participation measure. This fact reveals that the inclusion of the shortest paths in the link weight gives additional information, increasing the importance of those nodes that are in the way of the connection between other two. This kind of analysis is specially recommended for transport or communication networks, such as the airport network, where the reduction of the number of steps between nodes is a crucial issue. In these kinds of networks, the position of the node in the network, and its role in the connection with other nodes, can be more important than the degree itself.

Finally, we go one step further and analyze how a node distributes its connections among the communities. This information is included in the value of $p_{i}^{b}$, in the sense that, the more distributed the shortest paths are between the set of communities, the higher the value of $p_{i}^{b}$. Nevertheless, $p_{i}^{b}$ averages the contribution among all communities and does not allow to detect the communities where a given node $i$ is participating the most. To overcome this drawback, we can plot the contributions that a node makes to each community in a plot similar to Fig. 5. In this figure, we show the $\left(p_{i}^{b}, z_{i}^{c}\right)$ phase space of the fifteen nodes with the largest number of shortest paths. Nodes sizes are proportional to the number of shortest paths and colors inside each node indicate the community that is receiving the shortest paths. With this figure, we can see the nodes with higher relevance inside its community, their participation coefficients and how they participate in each community. It is worth noting that Anchorage Airport (Alaska) is the one with the highest number of shortest paths and, in addition, is one of the airports with higher relevance within its community and, at the same time, high participation coefficient. On the contrary, the shortest path connections of the Bethel Airport are only distributed inside its community, as indicated by a high $z_{i}^{c}$ combined with a low $p_{i}^{b}$. Finally, airports like Minneapolis-St. Paul have a complementary role, since they have a very high participation coefficient

Table 2. Variations of the roles of the nodes of the Airport Network of the USA [Newman, 2004]. Airports are ordered by the ranking of the community relevance $z_{i}^{b}$ (column 2) and betweenness participation coefficient $p_{i}^{b}$ (column 4 ). Note that the increase/decrease in the participation coefficient is much higher than in the community relevance, which has only small variations.

\begin{tabular}{|c|c|c|c|c|}
\hline Rank & $z_{i}^{b}$ (Community) & $\begin{array}{c}z_{i}^{b} \text { Rank } \\
\text { Improvement }\end{array}$ & $p_{i}^{b}$ (Community) & $\begin{array}{c}p_{i}^{b} \text { Rank } \\
\text { Improvement }\end{array}$ \\
\hline 1 & Anchorage Intl (C5) & +1 & Salt Lake City Intl (C1) & +16 \\
\hline 2 & Dallas/Fort Worth Intl (C1) & -1 & Minneapolis-St. Paul Intl/Wold- (C2) & +9 \\
\hline 3 & Chicago O'hare Intl (C2) & $=$ & Spokane Intl (C1) & +1 \\
\hline 4 & Stapleton Intl (C1) & $=$ & Seattle-Tacoma Intl (C1) & +9 \\
\hline 5 & Pittsburgh Intl (C2) & $=$ & Missoula Intl (C4) & +4 \\
\hline 6 & Bethel (C5) & 3 & Billings Logan Intl (C4) & +4 \\
\hline 7 & San Francisco Intl (C1) & -1 & Gallatin Field (C4) & -1 \\
\hline 8 & Charlotte/Douglas Intl (C2) & -1 & Anchorage Intl (C5) & +24 \\
\hline 9 & William B Hartsfield Atlan (C2) & -1 & Los Angeles Intl (C1) & +7 \\
\hline 10 & Lambert-St. Louis Intl (C2) & +1 & Detroit Metrop. Wayne Cou (C2) & +54 \\
\hline
\end{tabular}




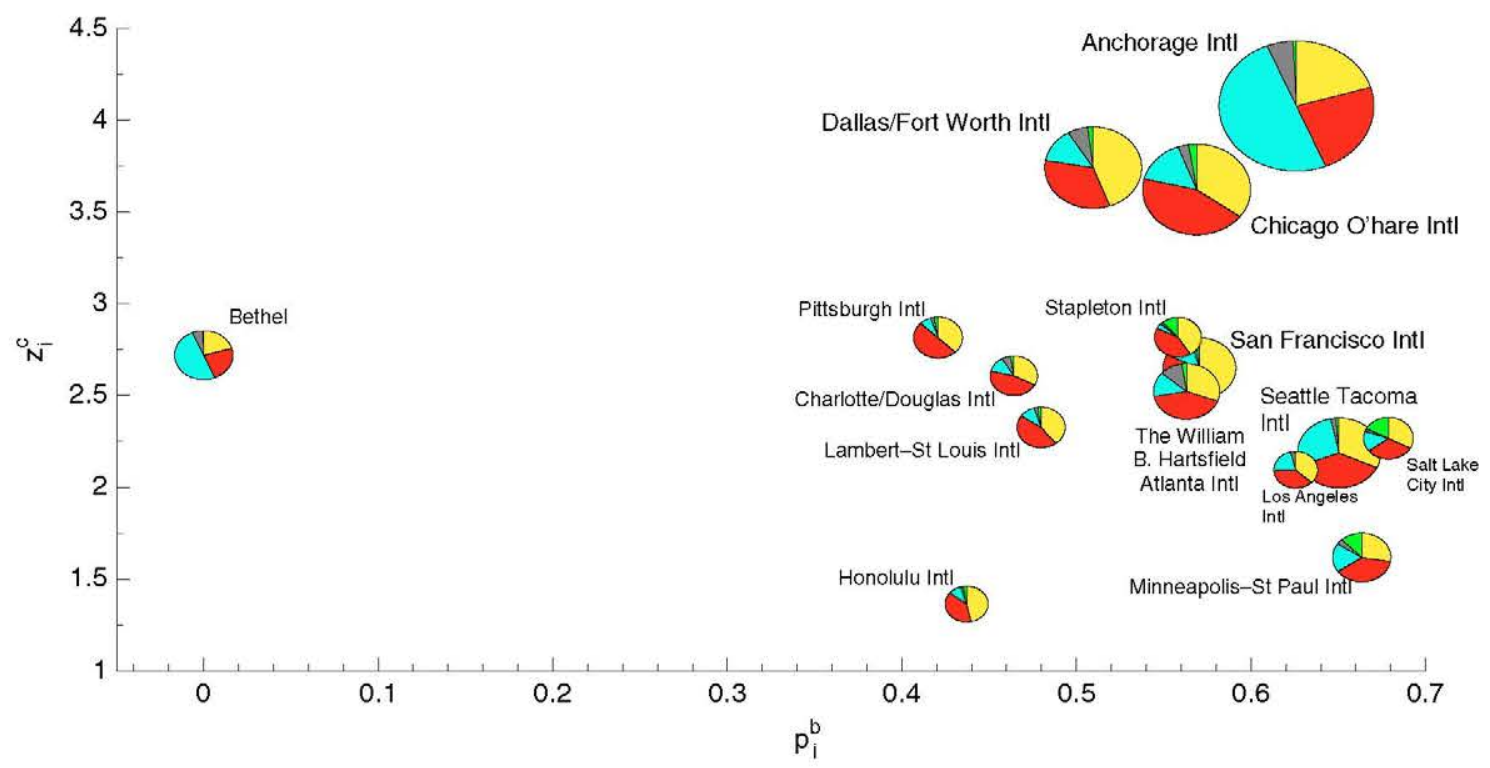

Fig. 5. Two-dimensional plot showing the $\left(p_{i}^{b}, z_{i}^{c}\right)$ phase space of the fifteen nodes with the largest number of shortest paths. Node sizes are proportional to the total number of shortest paths crossing the node. Colors inside nodes indicate the percentage of participation inside each community, specifically: C1 (yellow), C2 (red), C3 (grey), C4 (green) and C5 (light blue).

(connector with other communities) but a low local relevance, or at least, not too high when compared with other network hubs.

\section{Conclusions}

We have proposed a new methodology to evaluate the role that a node plays in the community structure of a network. We propose to evaluate the relevance of a node inside its community and the participation in other communities of the network by taking into account the number of shortest paths that pass through the node. In this way, we define a community $z$-score $z_{i}^{c}$ based on the closeness of a node inside its community. Next, we redefine the participation coefficient proposed by Guimerà et al. [Guimerà \& Amaral, 2005] and we weight the links with their number of shortest paths. Although both new parameters, $z_{i}^{c}$ and $p_{i}^{b}$, have a high computational cost $[O(N(M+N \log (N)))]$, they include information about the global structure of the network, while the previously proposed measures were based on local properties (node degree). We give some examples of how the role of a node changes when the new parameters are used, showing that the participation coefficient is specially affected when taking into account the shortest paths. Finally, we focus on the analysis of the American Airport Network [Newman, 2004], providing a new representation (see Fig. 5) of how the inter/intra community relevance of a node can be plotted. Despite previously proposed methods of role analysis being good approximations, we believe that this new methodology will give more accurate results, specially in the framework of communication and transportation networks.

\section{Acknowledgments}

This work was supported by the Spanish Ministry of S\&T [FIS2009-07072] and by the Community of Madrid under project URJC-CM-2010-CET-5006 and the R\&D Program of activities MODELICOCM [S2009ESP-1691].

Almendral, J. A., Leyva, I., Daqing, L., Sendiña-Nadal, I., Havlin, S. \& Boccaletti, S. [2010] "Dynamics of overlapping structures in modular networks," Phys. Rev. E 82, 016115.

Almendral, J. A., Criado, R., Leyva, I., Buldú, J. M. \& Sendiña-Nadal, I. [2011] "Introduction to focus issue: Mesoscales in complex networks," Chaos 21, 016101.

Arenas, A., Borge-Holthoefer, J., Gómez, S. \& ZamoraLopez, G. [2010] "Optimal map of the modular structure of complex networks," New J. Phys. 12, 053009 .

Boccaletti, S., Latora, V., Moreno, Y., Chavez, M. \& Hwang, D.-U. [2006] "Complex networks: Structure and dynamics," Phys. Rep. 424, 175-308. 
Buldú, J. M., Bajo, R., Maestú, F., Castellanos, N. Leyva, I., Gil, P., Sendiña-Nadal, I., Almendral, J. A., Nevado, A., del Pozo, F. \& Boccaletti, S. [2011] "Reorganization of functional networks in mild cognitive impairment," PLOS ONE 6, e19584.

Chavez, M., Hwang, D.-U., Amann, A., Hentschel, H. G. E. \& Boccaletti, S. [2005] "Synchronization is enhanced in weighted complex networks," Phys. Rev. Lett. 94, 218701.

Costa, L. F., Rodrigues, F. A., Travieso, G. \& Boas, P. R. V. [2007] "Characterization of complex networks: A survey of measurements," Adv. Phys. 56, 167-242.

Costa, L. F., Oliveira, O. N., Travieso, G., Rodrigues, F. A., Villas Boas, P. R., Antiqueira, L., Viana, M. P. \& da Rocha, L. E. C. [2011] "Analyzing and modeling real-world phenomena with complex networks: A survey of applications," Adv. Phys. 60, 329-412.

Fortunato, S. [2010] "Community detection in graphs," Phys. Rep. 486, 75-174.

Gross, T. \& Blasius, B. [2008] "Adaptive coevolutionary networks: A review," J. R. Soc. Interf. 6, 259-271.

Guimerà, R. \& Amaral, L. A. N. [2005] "Functional cartography of complex metabolic networks," Nature 433, 895-900.

Guimerà, R., Mossa, S., Turtschi, A. \& Amaral, L. A. N. [2005] "The worldwide air transportation network: Anomalous centrality, community structure, and cities' global roles," Proc. Natl. Acad. Sci. USA 102, 7794-7799.

Hagmann, P., Cammoun, L., Gigandet, X., Meuli, R., Honey, C. J., Wedeen, V. J. \& Sporns, O. [2008]
"Mapping the structural core of human cerebral cortex," PLoS Biol. 1, e159.

Krebs, V. [2011] "Social network analysis software \& services for organizations, communities, and their consultants," http://www.orgnet.com.

Lusseau, D. \& Newman, M. E. J. [2004] "Identifying the role that animals play in their social networks," Proc. R. Soc. London, Ser. B 27, 477-481.

Moon, S., Jinyoung, Y., Haewoon, K., Kim, D. \& Hawoong, J. [2010] "Understanding topological mesoscale features in community mining," Second Int. Conf. Communication Systems and Networks (COMSNETS), 2010, pp. 1-10.

Motter, A. E., Zhou, C. \& Kurths, J. [2005] "Enhancing complex-network synchronization," Europhys. Lett. 69, 334 .

Newman, M. E. J. [2003] "The structure and function of complex networks," SIAM Rev. 45, 67-256.

Newman, M. E. J. [2004] "Fast algorithm for detecting community structure in networks," Phys. Rev. E 69, 066133 .

Nishikawa, T., Motter, A. E., Lai, Y.-C. \& Hoppensteadt, F. C. [2003] "Heterogeneity in oscillator networks: Are smaller worlds easier to synchronize?" Phys. Rev. Lett. 91, 014101.

Teitelbaum, T., Balenzuela, P., Cano, P. \& Buldú, J. M. [2008] "Community structures and role detection in music networks," Chaos 18, 043105.

Zachary, W. W. [1977] "An information flow model for conflict and fission in small groups," J. Anthropol. Res. 33, 452-473. 